Pacific Journal of Mathematics

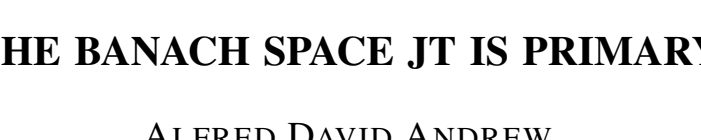




\title{
THE BANACH SPACE JT IS PRIMARY
}

\author{
A. D. ANDREW
}

It is proved that for every bounded linear operator $U$ on the James' tree space $\mathrm{JT}$ there is a subspace $X \subset \mathrm{JT}$, isometric to $\mathrm{JT}$, such that either $U$ or $(I-U)$ acts isomorphically on $X$ and either $U X$ or $(I-U) X$ is complemented in JT. As a consequence, JT is primary.

1. In this paper we prove that the James' tree space, JT, is primary. A Banach space $X$ is primary if whenever $X=Y \oplus Z$, either $Y$ or $Z$ is itself isomorphic to $X$. Many of the classical Banach spaces are known to be primary [1], [2], [3], [4], [5], [9], [10], [13].

The space JT was constructed by R. C. James [8] as an example of a separable space not containing $l_{1}$ yet having non-separable dual. It has also been studied by Lindenstrauss and Stegall [11]. Every subspace of JT contains $l_{2}$ [8], and JT has many subspaces isometric to the quasireflexive Banach space $J$ [6], [7]. Here we take the norm on $J$ to be

$$
\left\|\sum_{n=1}^{\infty} a_{n} x_{n}\right\|=\sup _{p_{1}<\cdots<p_{n}}\left\{\sum_{i=1}^{n-1}\left(\sum_{p_{i}+1}^{p_{i+1}} a_{i}\right)^{2}\right\}^{1 / 2} .
$$

To show that JT is primary, we prove that for each bounded linear operator $U$ on JT, there exists a subspace $X$ such that $U$ (or $I-U$ ) acts as an isomorphism on $X, X$ is isometric to $\mathrm{JT}$, and $U X$ (or $(I-U) X$ ) is complemented in JT. The space $X$ consists of functions supported on a certain subtree of the usual dyadic tree. The first part of the argument is a modification of an idea of Casazza and Lin [4]. That is, that if $U$ is a bounded linear operator on a space $Y$ with Schauder basis $\left\{y_{n}\right\}$, then either $\left\langle y_{n}^{*}, U y_{n}\right\rangle \geq \frac{1}{2}$ for infinitely many indices or $\left\langle y_{n}^{*},(I-U) y_{n}\right\rangle \geq \frac{1}{2}$ for infinitely many $n$. This idea was used also in [2].

In $\$ 2$ we fix the terminology concerning trees and present some elementary propositions about JT and trees. In $\$ 3$ these are used to construct the subspace $X$ described above. Our notation is standard in Banach space theory, as may be found in [12]. If $A$ is a subset of a Banach space, we denote the closed linear span of $A$ by $[A]$. The greatest integer function is denoted by $[\cdot]$. Standard perturbation arguments concerning stability properties of Schauder bases (e.g., Proposition 1.a.9. of [12]) are used in several places. 
2. In this section we present the definitions and some properties of JT as well as propositions guaranteeing the existence of certain subtrees. We begin with terminology concerning trees.

The standard tree is $\mathcal{T}=\left\{(n, i): 0 \leq n<\infty, 0 \leq i<2^{n}\right\}$. The points $(n, i)$ are called nodes. We say that $(n+1,2 i)$ and $(n+1,2 i+1)$ are the successors of $(n, i)$. A segment is a finite set $S=\left\{t_{1}, t_{2}, \ldots, t_{n}\right\}$ of nodes such that for each $j, t_{j+1}$ is a successor of $t_{j}$. $\mathcal{T}$ is partially ordered by the relation $<$, with $t_{1}<t_{2}$ if and only if $t_{1} \neq t_{2}$ and there is a segment $S$ with first element $t_{1}$ and last element $t_{2}$. If $t_{1}<t_{2}$ we say $t_{2}$ is a follower of $t_{1}$, thus reserving the word "successor" as meaning "immediate follower." The set $\left\{(n, i): 0 \leq i<2^{n}\right\}$ is called the $n$th level of $\mathcal{T}$. We denote the level of a node $t$ by lev $(t)$. An $n$-branch is a totally ordered set $\left\{\left(m, l_{m}\right)\right\}_{m=n}^{\infty}$, and a branch is a set which is an $n$-branch for some $n$. A tree is a partially ordered set $\delta$ which is order isomorphic to $\widetilde{\tau}$. If $\delta$ and $\delta^{\prime}$ are trees with $\mathcal{S}^{\prime} \subset \mathcal{S}$, we say $\mathcal{S}^{\prime}$ is a subtree of $\mathcal{S}$. If $\mathcal{S}$ is a tree and $\psi$ : $\mathcal{S} \rightarrow \mathcal{T}$ is an order isomorphism, we may use $\psi$ to carry the above terminology from $\mathcal{T}$ to $\mathcal{S}$. In particular, for $s \in \mathcal{S}$, we $\operatorname{define} \operatorname{lev}_{\S}(s)=$ $\operatorname{lev}(\psi(s))$.

We now define the James' tree space. For each $t \in \mathcal{T}$, let

$$
x_{t}(s)= \begin{cases}1, & t=s \\ 0, & t \neq s\end{cases}
$$

JT is the closed linear span of $\left\{x_{t}\right\}_{t \in \mathcal{T}}$ with respect to the norm

$$
\left\|\sum a_{n, i} x_{n, i}\right\|=\sup _{S_{1}, \ldots, S_{k}}\left\{\sum_{j=1}^{k}\left(\sum_{(n, i) \in S_{j}} a_{n, i}\right)^{2}\right\}^{1 / 2},
$$

where the supremum is taken over all finite collections of mutually disjoint segments $S_{1}, \ldots, S_{k}$. The elements $\left\{x_{n, i}\right\}$, in the order $x_{0,0}, x_{1,0}$, $x_{1,1}, x_{2,0}, x_{2,1}, x_{2,2}, x_{2,3}, \ldots$ form a boundedly complete basis for JT. We denote the sequence of biorthogonal functionals by $\left\{x_{n, i}^{*}\right\}$, and shall use the linear functionals and projections defined in the following formulas. Each is easily seen to have norm one. In these definitions, $S$ is a segment, $B$ a branch, $t$ a node, and $N$ an integer.

$$
\begin{aligned}
\left\langle f_{S}, x\right\rangle & =\sum_{t \in S}\left\langle x_{t}^{*}, x\right\rangle, \\
\left\langle f_{B}, x\right\rangle & =\sum_{t \in B}\left\langle x_{t}^{*}, x\right\rangle, \\
P_{S} x & =\sum_{t \in S}\left\langle x_{t}^{*}, x\right\rangle x_{t},
\end{aligned}
$$




$$
\begin{aligned}
P_{B} x & =\sum_{t \in B}\left\langle x_{t}^{*}, x\right\rangle x_{t}, \\
P_{N} x & =\sum_{\substack{t \\
\operatorname{lev}(t) \leq N}}\left\langle x_{t}^{*}, x\right\rangle x_{t}, \\
P_{t} x & =\sum_{s \geq t}\left\langle x_{s}^{*}, x\right\rangle x_{s} .
\end{aligned}
$$

The argument that shows JT to be primary is based on several propositions concerning trees and operators on JT.

Proposition 1. (a) For any subtree $\mathcal{S}$ of $\mathcal{T},\left[\left\{x_{t}: t \in \mathcal{S}\right\}\right]$ is isometric to $\mathrm{JT}$ and complemented in JT.

(b) For any branch $B \subset \mathcal{T}, P_{B} \mathrm{JT}$ is isometric to $J$.

Proof. Part (b), and the fact that $\left[\left\{x_{t}: t \in \delta\right\}\right]$ is isometric to JT follow directly from the definition of the norm in JT. Let $\left\{S_{t}\right\}_{t \in \delta}$ be a tree-like collection of disjoint segments of $\mathcal{T}$ such that $t \in \mathcal{S} \Rightarrow t \in S_{t}$ and such that there are no gaps in $\cup_{t \in \delta} S_{t}$. By this we mean that if $t_{1}, t_{2} \in \mathcal{S}$, and if $t_{2}$ is a successor in $\mathcal{S}$ of $t_{1}$, then whenever $t \in \mathcal{T}$ satisfies $t_{1}<t<t_{2}$, either $t \in S_{t_{1}}$, or $t \in S_{t_{2}}$. Then $\left[\left\{x_{t}: t \in \delta\right\}\right]$ is complemented by the norm one projection

$$
P x=\sum_{t \in \mathcal{S}}\left\langle f_{S_{t}}, x\right\rangle x_{t}
$$

Proposition 2. Let $U: \mathrm{JT} \rightarrow \mathrm{JT}$ be a bounded linear operator, $\varepsilon>0, N$ an integer, $\mathcal{S}$ a subtree of $\mathcal{T}$ and $t_{0} \in \mathcal{S}$. Then there exists $t_{1} \in \mathcal{S}, t_{1}>t_{0}$, such that

$$
\left\|P_{N} U x_{t_{1}}\right\|<\varepsilon
$$

Proof. If no such $t_{1}$ exists, then for any follower $t \in \mathcal{S}$ of $t_{0}$, there exists $t^{\prime}, \operatorname{lev}\left(t^{\prime}\right) \leq N$ with

$$
\left|\left\langle x_{t^{\prime}}^{*}, P_{N} U x_{t}\right\rangle\right| \geq \varepsilon / K,
$$

where $K=2^{N+1}-1$. Thus, for any $L$ and any collection $\left\{t_{l}\right\}_{l=1}^{L}$ of followers in $\delta$ of $t_{0},[L / K]$ of the $t_{l}$ satisfy (2) for the same node $t^{\prime}$. Hence there is a choice of signs $\left\{\theta_{l}= \pm 1\right\}$ such that

$$
\left\|\sum_{l=1}^{L} P_{N} U\left(\theta_{l} x_{t_{l}}\right)\right\| \geq\left\langle x_{t^{\prime}}^{*}, \sum_{l=1}^{L} P_{N} U\left(\theta_{l} x_{t_{l}}\right)\right\rangle \geq \frac{\varepsilon}{K}\left[\frac{L}{K}\right] .
$$


However, we may choose $\left\{t_{l}\right\}_{l=1}^{L}$ to be mutually non-comparable with respect to the order on $\mathcal{T}$, in which case it follows from (1) that

$$
\left\|\sum_{l=1}^{L} P_{N} U\left(\theta_{l} x_{t_{l}}\right)\right\| \leq\|U\|\left\|\sum \theta_{l} x_{t_{l}}\right\|=\|U\| L^{1 / 2}
$$

Since (3) and (4) are contradictory for large $L$, the proposition is proven.

Proposition 3. Let $U: \mathrm{JT} \rightarrow \mathrm{JT}$ be a bounded linear operator, $\varepsilon>0, N$ an integer, $\delta$ a subtree of $\mathcal{T}$ and $t_{0}, t_{1}, \ldots, t_{k}$ mutually incomparable nodes of $\mathcal{S}$. Then there exists $t>t_{0}, t \in \mathcal{S}, M \in N$, and segments $S_{0}, S_{1}, \ldots, S_{k}$ of $\widetilde{T}$ such that

(a) $\left\|P_{N} U x_{t}\right\|<\varepsilon$

(b) $\left\|\left(I-P_{M}\right) U x_{t}\right\|<\varepsilon$,

(c) For each $i, t_{i} \in S_{i}, S_{i}$ ends at level $M+1$ of $\mathcal{T}$, and there exists $t_{i}^{\prime} \in \mathcal{S}$ with $t_{i}^{\prime}>s$ for all $s \in S_{i}$,

(d) For each $i,\left\|P_{S_{i}} U x_{t}\right\|<\varepsilon$.

Proof. Let $K$ satisfy $2^{-K / 2}\|U\|<\varepsilon / 2$, and choose $N_{1} \geq$ $\max \left(N, \operatorname{lev}\left(t_{i}\right)\right)$ so that for each $i$, there are $2^{K}$ branches of $\mathcal{S}$ which pass through $t_{i}$ and through distinct nodes on the $N_{1}$ th level of $\sigma$. By Proposition 2, there exists $t>t_{0}, t \in \delta$ with $\left\|P_{N_{1}} U x_{t}\right\|<\varepsilon / 2$. Thus (a) is satisfied. Select $M>N_{1}$ so that (b) holds, and for each $i$, let $S_{i}^{1}, S_{i}^{2}, \ldots, S_{i}^{2^{K}}$ be segments of $\sigma_{\text {containing }} t_{i}$, passing through distinct nodes of the $N_{1}$ th level of $\mathcal{T}$ and satisfying (c).For each fixed $i$, we claim there exists $j$ so that

$$
\left\|P_{S_{i}^{j}}\left(I-P_{N_{1}}\right) U x_{t}\right\|<\varepsilon / 2 \text {. }
$$

Indeed, if this is not the case, then

$$
\frac{\varepsilon^{2}}{4} 2^{K} \leq \sum_{j=1}^{2^{K}}\left\|P_{S_{i}^{\prime}}\left(I-P_{N_{1}}\right) U x_{t}\right\|^{2} \leq\left\|\left(I-P_{N_{1}}\right) U x_{t}\right\|^{2} \leq\|U\|^{2} \leq \frac{\varepsilon^{2}}{4} 2^{K},
$$

a contradiction. Denoting this $S_{i}^{j}$ by $S_{i}$, we obtain

$$
\begin{aligned}
\left\|P_{S_{i}} U x_{t}\right\| & \leq\left\|P_{S_{t}}\left(I-P_{N_{1}}\right) U x_{t}\right\|+\left\|P_{S_{i}} P_{N_{1}} U x_{t}\right\| \\
& \leq \varepsilon / 2+\left\|P_{N_{1}} U x_{t}\right\|<\varepsilon .
\end{aligned}
$$

We omit the proofs of the next two propositions. Proposition 5 may be proved inductively, using Proposition 4 repeatedly.

Proposition 4. Let $\delta$ be a tree and $A$ a subset of $\mathcal{S}$. Then there exists a subtree $\delta^{\prime} \subset \mathcal{S}$ such that either $\delta^{\prime} \subset A$ or $\delta^{\prime} \subset \tilde{A}$, the complement of $A$. 
Proposition 5. Let $f$ be a bounded real valued function defined on a tree $\mathcal{S}$. Then for any $\varepsilon>0$, there exists a subtree $S^{\prime}$ such that for any branch $B$ of $\mathcal{S}^{\prime}$

(a) $\lim _{t \rightarrow \infty ; t \in B} f(t)=L_{B}$ exists, and

(b) $\Sigma_{t \in B}\left|f(t)-L_{B}\right|<\varepsilon$.

3. In this section we apply the results of $\$ 2$ to prove

THEOREM 6. Let $U$ be a bounded linear operator on JT. Then there exists a subspace $X$ of $\mathrm{JT}$ such that

(a) $X$ is isometric to JT,

(b) $\left.U\right|_{X}\left(\left.\operatorname{or}(I-U)\right|_{X}\right)$ is an isomorphism,

(c) $U X($ or $(I-U) X)$ is complemented in JT.

Proof. We will construct a subtree $\mathcal{S} \subset \mathcal{T}$ such that either $\left\{U x_{t}\right\}_{t \in \mathcal{S}}$ or $\left\{(I-U) x_{t}\right\}_{t \in \delta}$ is equivalent to $\left\{x_{t}\right\}$ and has complemented span. The desired subspace is then $X=\left[\left\{x_{t}\right\}_{t \in \delta}\right]$.

Let $V=I-U$ and $0<\gamma<\frac{1}{2}$. For each $t \in \mathcal{T}$, let $B_{t}$ be a 0 -branch containing $t$. Then

$$
1=\left\langle f_{B_{t}}, U x_{t}\right\rangle+\left\langle f_{B_{t}}, V x_{t}\right\rangle,
$$

so either $\left\langle f_{B_{t}}, U x_{t}\right\rangle \geq \frac{1}{2}$ or $\left\langle f_{B_{t}}, V x_{t}\right\rangle \geq \frac{1}{2}$. By standard perturbation arguments we may assume $U x_{t}$ and $V x_{t}$ are finitely supported, say that $P_{N_{t}} U x_{t}=U x_{t}$ and $P_{N_{t}} V x_{t}=V x_{t}$. Denoting by $S_{t}$ the segment $B_{t} \cap\{s$ : $\left.\operatorname{lev}(s) \leq N_{t}\right\}$, we may assume that for each $t$, either $\left\langle f_{S_{t}}, U x_{t}\right\rangle>\gamma$ or $\left\langle f_{S_{t}}, V x_{t}\right\rangle>\gamma$. Denote the last element of $S_{t}$ by $l(t)$.

We construct a subtree $\delta_{1} \subset \mathcal{T}$ inductively. Let $(0,0) \in \delta_{1}$, and assume the $n$th level of $\delta_{1}$ is already constructed. The $(n+1)$ st level of $\delta_{1}$ consists of all nodes in $\mathcal{T}$ which are successors of nodes $l(t)$ where $t$ belongs to the $n$th level of $\mathcal{S}_{1}$.

Let $A=\left\{t \in \mathcal{S}_{1}:\left\langle f_{S_{t}}, U x_{t}\right\rangle>\gamma\right\}$. By Proposition 4 there is a subtree $\delta_{2}$ of $\mathcal{S}_{1}$ such that either $\mathcal{S}_{2} \subset A$ or $\mathcal{S}_{2} \subset \tilde{A}$. We shall assume $\mathcal{S}_{2} \subset A$, and hence shall discuss the operator $U$, rather than $I-U$. For each $t \in \delta_{2}$, let $\gamma_{t}=\left\langle f_{S_{t}}, U x_{t}\right\rangle$. Then $\gamma \leq \gamma_{t} \leq\|U\|$, so by Proposition 5 we may assume that for each branch $B$ of $\delta_{2}$

$$
\lim _{\substack{B \rightarrow \infty \\ B}} \gamma_{t}=\gamma_{B} \text { exists, }
$$

and

$$
\sum_{t \in B}\left|\gamma_{t}-\gamma_{B}\right|<\gamma / 2
$$


Condition (6) ensures that the multiplier operator $T$ on $J$ defined by $T x_{t}=\left(\gamma_{t} / \gamma_{B}\right) x_{t}$ satisfies $\|I-T\|<\frac{1}{2}$. Hence $T$ is invertible and $\left\|T^{-1}\right\|$ $<2$.

The desired subtree $\delta=\{t(n, i)\} \subset \mathcal{S}_{2}$ is constructed inductively using Proposition 3. We will not reproduce the full details, but will indicate the first step. Parts (a), (b) and (d) of Proposition 3 are "gliding hump" conclusions, and allow us to compute norms. Part (c) guarantees that the inductive construction may be continued in $\delta_{2}$.

Let $\varepsilon>0$, and $\left\{\varepsilon_{t}>0\right\}$ a sequence such that $\sum \varepsilon_{i}<\varepsilon$. Let $t(0,0)$ be the initial node of $\mathcal{S}_{2}$, place $t(0,0) \in \mathcal{S}$, and let $N$ be an integer such that

$$
\left(I-P_{N}\right) U x_{t(0,0)}=0 \text {. }
$$

By Proposition 3, there exists $t(1,0)>l(t(0,0)), t(1,0) \in \mathcal{S}_{2}$, an integer $M$ and a segment $S_{0}$, with $l(t(0,0)) \in S_{0}$ satisfying

$$
\begin{array}{r}
\left\|P_{N} U x_{t(1,0)}\right\|<\varepsilon_{1}, \\
\left\|\left(I-P_{M}\right) U x_{t(1,0)}\right\|<\varepsilon_{2},
\end{array}
$$

and

$$
\left\|P_{S_{0}} U x_{t(1,0)}\right\|<\varepsilon_{3} .
$$

Let $t(1,0) \in \mathcal{S}$. Now let $t_{0}$ be a node of $\mathcal{S}_{2}$ following $S_{0}$, and $t_{1}$ a node of $\mathcal{S}_{2}$ following $S_{t(1,0)}$. Again by Proposition 3, there exists $t(1,1) \in \mathcal{S}_{2}$, $t(1,1)>t_{0}$, an integer $M^{\prime}$ and a segment $S_{1}$ following $t_{1}$ so that

$$
\begin{array}{r}
\left\|P_{M} U x_{t(1,1)}\right\|<\varepsilon_{4}, \\
\left\|\left(I-P_{M^{\prime}}\right) U x_{t(1,1)}\right\|<\varepsilon_{5},
\end{array}
$$

and

$$
\left\|P_{S_{1}} U x_{t(1,1)}\right\|<\varepsilon_{6} .
$$

The first level of $\delta$ is completed by placing $t(1,1) \in \mathcal{S}$.

Proceeding in this fashion, after standard perturbation arguments, we may assume that for each $t \in \mathcal{\delta}$,

$$
\begin{aligned}
& \left\langle f_{S_{t(n+1, i)}}-f_{S_{l(n, l t / 2])}, U x_{t}}\right\rangle \\
& = \begin{cases}\gamma_{t(n+1, i)}, & t=t(n+1, i), \\
0 & \text { otherwise. }\end{cases}
\end{aligned}
$$


With each $t \in \mathcal{S}$, we associate a segment $S_{t}^{\prime}$ passing through $t$ and the support of $U x_{t}$. The $S_{t(n, j)}^{\prime}$ are constructed in pairs as follows. Let $t_{1}$ be the last node of $\mathcal{T}$ belonging to $S_{t(n, 2 i)} \cap S_{t(n, 2 i+1)}, t_{2}$ the last node of $\mathcal{T}$ in $S_{t(n+1,4 i)} \cap S_{t(n+1,4 i+1)}$, and $t_{3}$ the last node of $\mathcal{T}$ in $S_{t(n+1,4 i+2)} \cap$ $S_{t(n+1,4 i+3)}$. Let $S_{t(n, 2 i)}^{\prime}$ be the maximal segment with last element $t_{2}$ and not containing $t_{1}$, and let $S_{t(n, 2 i+1)}^{\prime}$ be the maximal segment having last element $t_{3}$ and not containing $t_{1}$. Then there are no gaps (in $\mathcal{T}$ ) between the $S_{t}^{\prime}$, and by (7)

$$
\left\langle f_{S_{t(n, t)}^{\prime}}, U x_{t}\right\rangle= \begin{cases}\gamma_{t(n, i)}, & t=t(n, i), \\ 0 & \text { otherwise. }\end{cases}
$$

To show that $\left\{U x_{t}\right\}_{t \in \delta}$ is equivalent to $\left\{x_{t}\right\}_{t \in S}$, let $\left\{a_{n, i}\right\}$ be a finite set of scalars. There exist segments $S_{1}, \ldots, S_{k}$ such that

$$
\left\|\sum a_{n, i} x_{t(n, i)}\right\|=\left\{\sum_{j=1}^{k}\left(\sum_{S_{j}} a_{n, i}\right)^{2}\right\}^{1 / 2},
$$

and we may assume that each $S_{j}$ is a union of segments $S_{t}^{\prime}$. Furthermore, there exist disjoint branches $B_{1}, \ldots, B_{l}$ such that each $S_{j}$ is a subset of some $B_{i}$. Then

$$
\begin{aligned}
\left\|\sum a_{n, i} x_{t(n, i)}\right\| & =\left\{\sum_{j=1}^{l}\left(\sum_{S_{l} \subset B_{j}}\left(\sum_{S_{t}} a_{n, i}\right)^{2}\right)\right\}^{1 / 2} \\
& =\left\{\sum_{j}\left\|P_{B_{j}}\left(\sum a_{n, i} x_{t(n, i)}\right)\right\|_{j}^{2}\right\}^{1 / 2},
\end{aligned}
$$

by Proposition 1,

$$
\begin{aligned}
& \leq \frac{1}{\gamma}\left\{\sum_{j}\left\|P_{B_{j}}\left(\sum \gamma_{B_{j}} a_{n, i} x_{t(n, i)}\right)\right\|_{J}^{2}\right\}^{1 / 2}, \\
& \leq \frac{2}{\gamma}\left\{\sum_{j}\left\|P_{B_{J}}\left(\sum a_{n, i} \gamma_{t(n, i)} x_{t(n, i)}\right)\right\|_{J}^{2}\right\}^{1 / 2},
\end{aligned}
$$

by the remark following (6),

$$
=\frac{2}{\gamma}\left\{\sum_{j} \sum_{S_{i}^{\prime \prime} \subset B_{j}}\left\{\sum_{S_{i}^{\prime \prime}} a_{n, i} \gamma_{t(n, i)}\right\}^{2}\right\}^{1 / 2},
$$


for some choice of disjoint segment $S_{i}^{\prime \prime}$ containing the $S_{t}^{\prime}$,

$$
\leq \frac{2}{\gamma}\left\|\sum a_{n, i} U x_{t(n, i)}\right\|, \quad \text { by (7). }
$$

Thus for any scalar sequence $\left\{a_{n, i}\right\}$, we have

$$
\begin{aligned}
\left\|\sum a_{n, i} x_{t(n, i)}\right\| & \leq \frac{2}{\gamma}\left\|\sum a_{n, i} U x_{t(n, i)}\right\| \\
& \leq \frac{2\|U\|}{\gamma}\left\|\sum a_{n, i} x_{t(n, i)}\right\|,
\end{aligned}
$$

so that $\left\{x_{t(n, i)}\right\}$ and $\left\{U x_{t(n, i)}\right\}$ are equivalent. Thus $U$ acts as an isomorphism on $X=\left[\left\{x_{t}\right\}_{t \in \delta}\right]$ and $X$ is isometric to JT be Proposition 1.

To see that $\left[\left\{U x_{t}\right\}_{t \in \delta}\right]$ is complemented, let $P$ be the projection onto $\left[\left\{x_{t}\right\}_{t \in \delta}\right]$ defined in the proof of Proposition 1, using the segments $S_{t}^{\prime}$. The argument that shows that $\left\{x_{t}\right\}_{t \in \delta}$ and $\left\{U x_{t}\right\}_{t \in \delta}$ are equivalent also shows that $S=P \mid\left[\left\{U x_{t}\right\}_{t \in \delta}\right]$ is invertible. Then $\left[\left\{U x_{t}\right\}_{t \in \delta}\right]$ is complemented by $S^{-1} P$.

THEOREM 7. The James tree space JT is primary.

Proof. We use the Pelczynski decomposition method. Observe that with $B=\{(n, 0): n=0,1,2, \ldots\}$, we have

$$
\mathrm{JT}=P_{B} \mathrm{JT} \oplus\left(\sum_{n=1}^{\infty} \oplus P_{n, 1} \mathrm{JT}\right)_{l_{2}} \approx J \oplus\left(\sum_{n=1}^{\infty} \oplus \mathrm{JT}\right)_{l_{2}} .
$$

From this it follows that JT is isomorphic to its square, since

$$
\begin{aligned}
\mathrm{JT} & \approx J \oplus(\Sigma \oplus \mathrm{JT})_{l_{2}} \approx J \oplus \mathrm{JT} \oplus(\Sigma \oplus \mathrm{JT})_{l_{2}} \\
& \approx \mathrm{JT} \oplus J \oplus(\Sigma \oplus \mathrm{JT})_{l_{2}} \approx \mathrm{JT} \oplus \mathrm{JT} .
\end{aligned}
$$

Now, if $\mathrm{JT}=Y \oplus Z$, by Theorem 6 we may assume $Y \approx W \oplus \mathrm{JT}$. Then

$$
\begin{aligned}
Y & \approx W \oplus \mathrm{JT} \approx W \oplus \mathrm{JT} \oplus \mathrm{JT} \approx Y \oplus \mathrm{JT} \\
& \approx Y \oplus(\Sigma \oplus \mathrm{JT})_{l_{2}} \oplus J \\
& \approx Y \oplus\left(\sum \oplus Y \oplus Z\right)_{l_{2}} \oplus J \approx \mathrm{JT} .
\end{aligned}
$$




\section{REFERENCES}

[1] D. Alspach and Y. Benyamini, Primariness of spaces of continuous functions on ordinals, Israel J. Math., 27 (1977), 64-92.

[2] D. Alspach, P. Enflo, and E. Odell, On the structure of separable $\mathfrak{L}_{p}$ spaces $(1<p<$ $\infty)$, Studia Math., 60 (1977), 79-90.

[3] P. G. Casazza, James' quasi-reflexive space is primary, Israel J. Math., 26 (1977), 294-305.

[4] P. G. Casazza and B. L. Lin, Projections on Banach spaces with symmetric bases, Studia Math., 52 (1974), 189-193.

[5] P. G. Casazza, C. A. Kottman, and B. L. Lin, On some classes of primary Banach spaces, Canad. J. Math., 29 (1977), 856-873.

[6] R. C. James, Bases and reflexivity of Banach spaces, Ann. Math., 52 (1951), 518-527.

[7] A non-reflexive Banach space isometric with its second conjugate space, Proc. Nat. Acad. Sci. USA, 37 (1951), 174-177.

[8] A separable somewhat reflexive Banach space with non-separable dual, Bull. Amer. Math. Soc., 80 (1974), 738-743.

[9] J. Lindenstrauss, On complemented subspaces of $m$, Israel J. Math., 5 (1967), $153-156$.

[10] J. Lindenstrauss and A. Pelczynski, Contributions to the theory of classical Banach spaces, J. Functional Anal., 8 (1971), 225-249.

[11] J. Lindenstrauss and C. Stegall, Examples of separable spaces which do not contain $l_{1}$ and whose duals are non-separable, Studia Math., 54 (1975), 81-105.

[12] J. Lindenstrauss and L. Tzafriri, Classical Banach Spaces I, Springer-Verlag, New York, 1977.

[13] A. Pelczynski, Projections in certain Banach spaces, Studia Math., 19 (1960), 209-228.

Received December 14, 1981 and in revised form April 9, 1982.

GeORgia InStitute of TeChNOLOGY

AtLanta, GA 30332 



\section{PACIFIC JOURNAL OF MATHEMATICS EDITORS}

Donald BABBITT (Managing Editor)

University of California

Los Angeles, CA 90024

Hugo Rossi

University of Utah

Salt Lake City, UT 84112

C. C. Moore and Arthur Ogus

University of California

Berkeley, CA 94720
J. DugundiI

Department of Mathematics

University of Southern California

Los Angeles, CA 90089-1113

R. Finn and H. SAMELSON

Stanford University

Stanford, CA 94305

ASSOCIATE EDITORS
R. ARENS
E. F. BECKENBACH
B. H. NeUmanN
F. WOLF
K. YosHIDA (1906-1982)

\section{SUPPORTING INSTITUTIONS}

UNIVERSITY OF ARIZONA

UNIVERSITY OF BRITISH COLUMBIA

CALIFORNIA INSTITUTE OF TECHNOLOGY

UNIVERSITY OF CALIFORNIA

MONTANA STATE UNIVERSITY

UNIVERSITY OF NEVADA, RENO

NEW MEXICO STATE UNIVERSITY

OREGON STATE UNIVERSITY
UNIVERSITY OF OREGON

UNIVERSITY OF SOUTHERN CALIFORNIA

STANFORD UNIVERSITY

UNIVERSITY OF HAWAII

UNIVERSITY OF TOKYO

UNIVERSITY OF UTAH

WASHINGTON STATE UNIVERSITY

UNIVERSITY OF WASHINGTON 


\section{Pacific Journal of Mathematics}

\section{Vol. 108, No. $1 \quad$ March, 1983}

Waleed A. Al-Salam and A. Verma, $q$-Konhauser polynomials $\ldots \ldots \ldots \ldots 1$

Alfred David Andrew, The Banach space JT is primary $\ldots \ldots \ldots \ldots \ldots . . .6$

Thomas E. Bengtson, Bessel functions on $P_{n} \ldots \ldots \ldots \ldots \ldots \ldots$

Joaquim Bruna Floris and Francesc Tugores, Free interpolation for

holomorphic functions regular to the boundary $\ldots \ldots \ldots \ldots \ldots \ldots \ldots \ldots$

Peter Dierolf and Susanne Dierolf, Topological properties of the dual pair

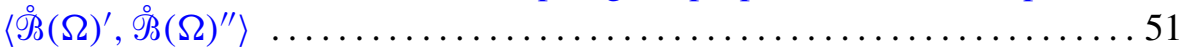

Gerald Arthur Edgar, An ordering for the Banach spaces $\ldots \ldots \ldots \ldots \ldots 83$

Basil Gordon, A proof of the Bender-Knuth conjecture . . . . . . . . . . . . . 99

Harold T. Hodes, A minimal upper bound on a sequence of Turing degrees

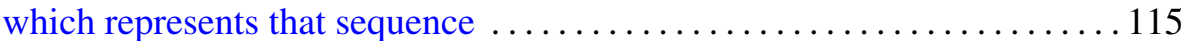

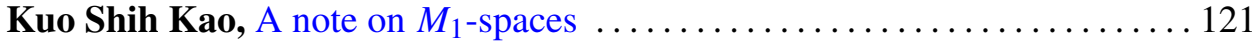

Frank Kost, Topological extensions of product spaces ................ 129

Eva Lowen-Colebunders, On the convergence of closed and compact

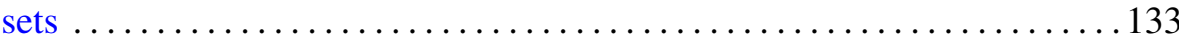

Doron Lubinsky, Divergence of complex rational approximations . . . . . . 141

Warren May and Elias Hanna Toubassi, Endomorphisms of rank one

mixed modules over discrete valuation rings $\ldots \ldots \ldots \ldots \ldots \ldots \ldots \ldots \ldots$

Richard Patrick Morton, The quadratic number fields with cyclic

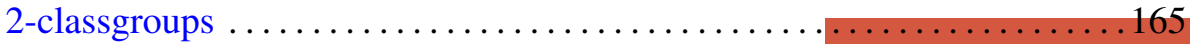

Roderic Murufas, Rank of positive matrix measures . . . . . . . . . . . . 177

Helga Schirmer, Fixed point sets of homotopies . . . . . . . . . . . . . 191

E. Taflin, Analytic linearization of the Korteweg-de Vries equation ........ 203

James Thomas Vance, Jr., $L^{p}$-boundedness of the multiple Hilbert

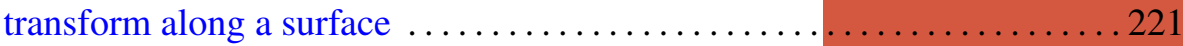

Hiroshi Yamaguchi, A property of some Fourier-Stieltjes transforms . . . . . 243 